\title{
POROELASTIC BEHAVIOUR OF THE DEGENERATING HUMAN INTERVERTEBRAL DISC: A TEN-DAY STUDY IN A LOADED DISC CULTURE SYSTEM
}

\author{
K.S. Emanuel ${ }^{1}$, P-P.A. Vergroesen ${ }^{1}$, M. Peeters ${ }^{1}$, R.M. Holewijn ${ }^{1}$, I. Kingma ${ }^{2}$ and T.H. Smit ${ }^{1, *}$ \\ ${ }^{1}$ Department of Orthopaedic Surgery, MOVE Research Institute Amsterdam, VU University Medical Centre, \\ Amsterdam, the Netherlands \\ ${ }^{2}$ MOVE Research Institute Amsterdam, Faculty of Human Movement Sciences, VU University Amsterdam, \\ Amsterdam, the Netherlands
}

\begin{abstract}
The intervertebral disc (IVD) allows flexibility to the vertebral column, and transfers the predominant axial loads during daily activities. Its axial biomechanical behaviour is poroelastic, due to the water-binding and releasing capacity of the nucleus pulposus. Degeneration of the intervertebral disc presumably affects both the instantaneous elastic response to the load on the IVD and the subsequent interstitial flow of fluid. This study aims to quantify the poroelastic behaviour of the IVD and its change with degeneration, as defined by the magnetic resonance imaging-based Pfirrmann Score (PS). For a period of ten days, 36 human lumbar IVDs were loaded with a simulated physiological axial loading regime, while deformation was monitored. The IVDs responded to the loads with instantaneous elastic and slow poroelastic axial deformation. Several mechanical parameters changed throughout the first five days of the experiment, until the IVDs settled into a dynamic equilibrium. In this equilibrium, degeneration was significantly related to a decrease in disc height loss during the daytime high load phase $(\rho=-0.49)$, and to a decrease in the rate of this deformation during the final half hour of each day $(\rho=-0.53)$. These properties were related to the nucleus glycosaminoglycan/hydroxyproline (GAG/HYP) ratio, rather than GAG content alone, indicating that remodelling of the extracellular matrix reduces poroelastic properties of the IVD. This implies that the degenerated discs have a reduced capacity to bind water and/or a reduced resistance against fluid flow. The resulting loss in hydrostatic pressure may further change cell behaviour in the nucleus pulposus.
\end{abstract}

Keywords: Intervertebral disc, degeneration, biomechanics, poroelastic behaviour, Pfirrmann, magnetic resonance imaging, glycosaminoglycan, biochemistry, loaded disc culture system, spine.

*Address for correspondence:

Theodoor H. Smit

Department of Orthopaedic Surgery

VU University Medical Centre

De Boelelaan 1117, $1081 \mathrm{HV}$, Amsterdam

the Netherlands

Telephone Number: +31-204442988

FAX Number: +31- 204442357

E-mail: th.smit@vumc.nl

\section{Introduction}

Low back pain (LBP) is one of the most frequent medical complaints in western society, with enormous socioeconomic impact (Katz, 2006). Lifetime prevalence of more than three months of LBP is $20 \%$ (Hoy et al., 2012), and direct and indirect costs of LBP are estimated at $0.6 \%$ of the gross national product in the Netherlands (Lambeek et al., 2011). Despite this, the scientific progress to improve prevention and cure has been limited so far (Balagué et al., 2012). Progress has been hampered, among others, by difficulties in pinpointing the aetiology of non-specific LBP (Andersson, 1999). In the past two decades, the relation between intervertebral disc (IVD) degeneration and LBP has been under debate (Adams et al., 2000; Balagué et al., 2012; van Tulder et al., 1997), but recent epidemiological studies show that IVD degeneration indeed is a significant predictor (Cheung et al., 2009; Livshits et al., 2011; Wang et al., 2012).

\section{Structure and function of the intervertebral disc}

The IVD is a "cushion-like structure" (Chan et al., 2011). Its core is the nucleus pulposus, a gel-like matrix rich in proteoglycans, which is contained by the annulus fibrosus, consisting of alternating lamellae of collagen type-I fibres. The IVD's function is essentially mechanical, and can be seen as an elastic hinge that provides flexibility and transfers loads in the vertebral column (Brinckmann and Grootenboer, 1991; Chan et al., 2011). The functional status of IVDs can therefore be described by considering their biomechanical properties. The predominant load on the IVD is axial compression (Smit et al., 1997). The response of the IVD to this load has been modelled as a system with poroelastic properties (O'Connell et al., 2011; van der Veen et al., 2013). The slow poroelastic behaviour is mediated by fluid flow, due to the binding and releasing of water by the proteoglycans in the nucleus. The bound water in the nucleus generates an intradiscal osmotic pressure, which separates the vertebrae and tensions the annulus fibres (Brinckmann and Grootenboer, 1991). Upon axial loading, there is an instantaneous elastic response, which is the result of the tensioning of annulus fibres (Johnson et al., 1982). At the same time, the axial pressure surpasses the intradiscal pressure and bound water is slowly lost from the IVD, resulting in a characteristic slow creep (Adams and Roughley, 2006). In healthy discs, the cells in the IVD produce the proteoglycans that generate its intradiscal osmotic pressure, and thereby maintain its biomechanical function. 
In degenerated intervertebral discs, a marked reduction in proteoglycan content as well as disorganisation of the annulus fibres has been observed (Adams and Roughley, 2006). This affects the water-binding capacity, thereby reducing the hydrostatic pressure (Sato et al., 1999), and altering the response to compressive loads (Hwang et al., 2012; Vergroesen et al., 2014). The influence of proteoglycan content on IVD mechanics has been shown indirectly, as enzymatic digestion of proteoglycans, induced by injection of chondroitinase ABC (CABC), caused disc narrowing (Fry et al., 1991; Hoogendoorn et al., 2007; Lü et al., 1997), and changed flexion mechanics (Lü et al., 1997) and intradiscal pressure (Sasaki et al., 2001). Furthermore, it was shown that nucleus pulposus material properties were moderately related to proteoglycan content (Johannessen and Elliott, 2005). However, the direct relation between proteoglycan content and whole-disc mechanics has to our knowledge not yet been studied. This relation however may be the core of the degeneration process, because the loss of proteoglycans and the related reduced hydrostatic pressure increases the shear stresses in the nucleus (Hwang et al., 2012). The increase in shear stresses in turn influences cell activity, as hydrostatic pressure leads to different gene expression in the cells than when subjected to shear stresses, the latter further reducing proteoglycan content (Carter and Wong, 2003; Hsieh and Twomey, 2010; Paul et al., 2013). Therefore, mechanical function of the IVD and disc degeneration are presumably closely related (Vergroesen et al., 2015a).

A common definition of IVD degeneration is based on the appearance of the IVD on magnetic resonance imaging (MRI). Many epidemiological studies have used this definition to investigate the relation between IVD degeneration and low-back pain (Cheung et al., 2009; Livshits et al., 2011; Luoma et al., 2000; van Tulder et al., 1997; Wang et al., 2012). The most commonly used grading method for MRI images is the algorithm introduced by Pfirrmann et al. (2001). In short, the signal intensity on T2-weighted MRI images is used to estimate water content, and, together with morphological parameters, this is used to grade for IVD degeneration on a scale from 1-5. It is considered "the most clinically relevant" (Kettler and Wilke, 2006) lumbar IVD degeneration grading method, because of its non-invasive nature and high reliability. However, the relation to the functional status of the tissue has been under debate, because the method was introduced without investigating the relation to tissue properties (Urban and Winlove, 2007).

To date, the relationship between mechanical function and degeneration has not been elucidated. Antoniou et al., (2013) recently reviewed five studies that correlate biomechanical properties of small samples of nucleus or annulus in vitro to either age or IVD degeneration grading; however, these correlations were weak, possibly due to the separation of nucleus and annulus. Other studies used complete motion segments for biomechanical in vitro research, but the IVDs used were removed from their physiological conditions and unloaded for a while, which may have influenced the results of the research performed so far (e.g. Hwang et al., 2012; Wilke et al., 1998). The recent development of animal disc culture systems may benefit research relating degeneration to mechanical function, as they allow extended loading of animal IVDs in order to approximate physiological conditions (Jim et al., 2011; Korecki et al., 2007; Paul et al., 2012). Besides creating a simulated physiological biomechanical environment, some systems can measure the disc's response to the loading, creating new opportunities to evaluate the functional status of IVDs.

In this study, the advantages of a loaded disc culture system are exploited to investigate the biomechanical properties of human IVDs during long-term loading, and its association with MRI-based degeneration grading. The application of long-term physiological loading can reduce the effect of the inevitable unloading prior to the experiment, and allows the IVDs to settle in a dynamic equilibrium, similar to the situation in vivo. Because degeneration affects the structures that determine the water-binding capacity of the IVD, we hypothesise that Pfirrmann Score correlates with biomechanical parameters that describe the poroelastic response to axial load. Furthermore, we hypothesise that a reduction in GAG content of the nucleus, a measure for proteoglycan content, is related to a reduced poroelastic response.

\section{Materials and Methods}

48 lumbar IVDs were obtained from 12 spines of freshly frozen human cadavers (age: 64-93 years). After thawing, MRI T2-weighted images of all spines were obtained using a Siemens ${ }^{\odot}$ Symphony $1.5 \mathrm{~T}$ scanner (Syngo MR A30, software NUMARIS/4, Berlin, Germany). Three observers independently graded the IVDs for degeneration using the Pfirrmann Score (PS) on a scale of 1-5 (Pfirrmann et al.,

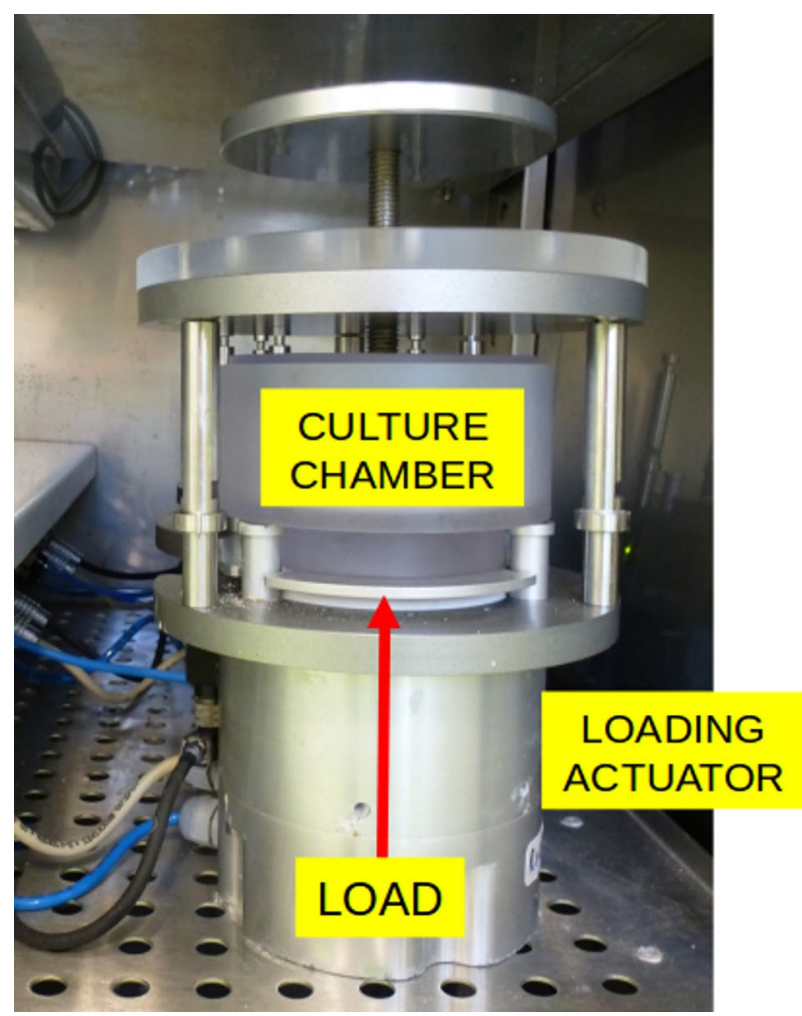

Fig. 1. The loaded disc culture system used in this study. The IVD is located inside the culture chamber. 


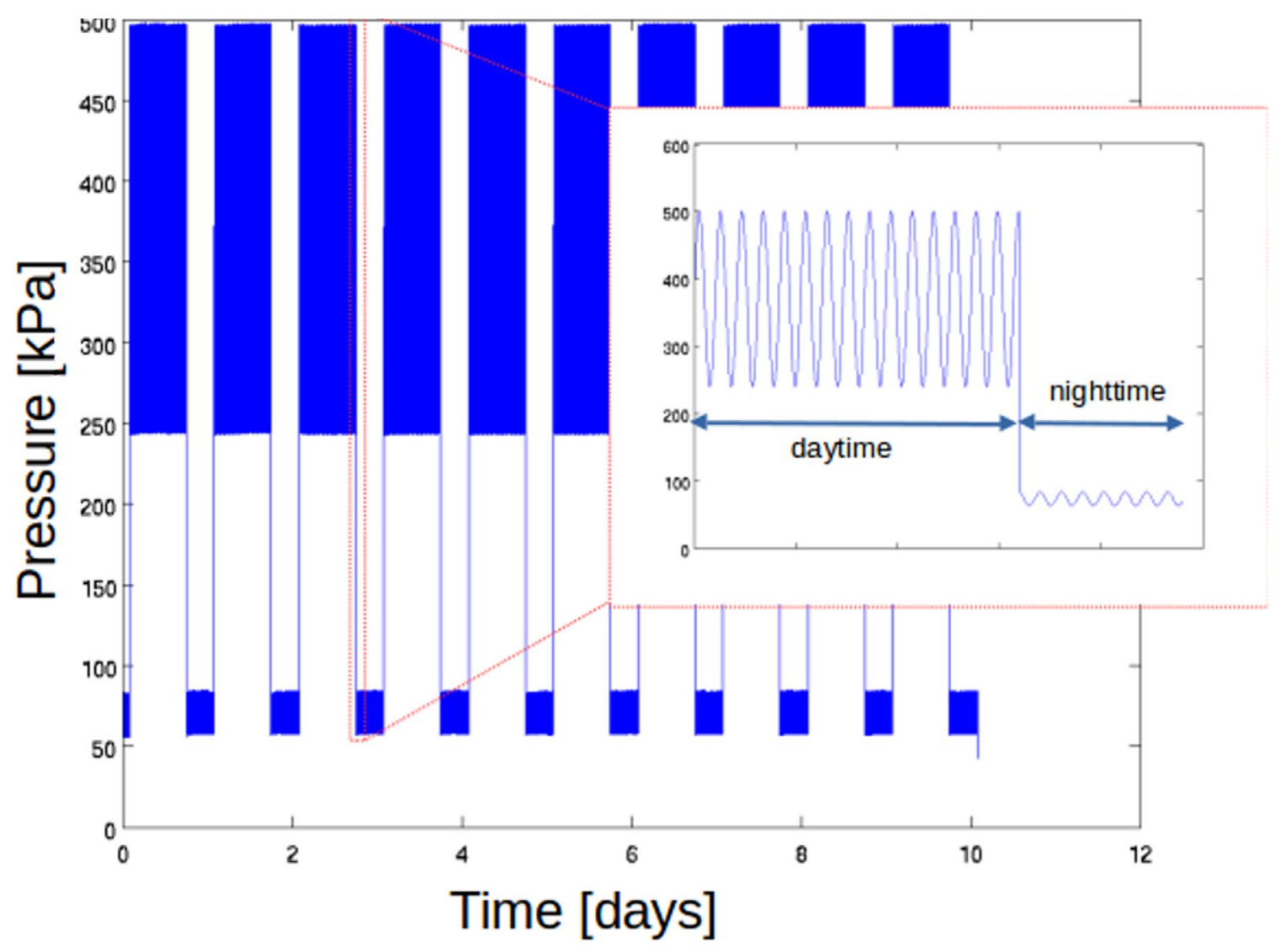

Fig. 2. Loading regime. Applied axial load on top of the IVD. A diurnal rhythm of $16 \mathrm{~h}$ high dynamic loading and $8 \mathrm{~h}$ low dynamic loading was applied.

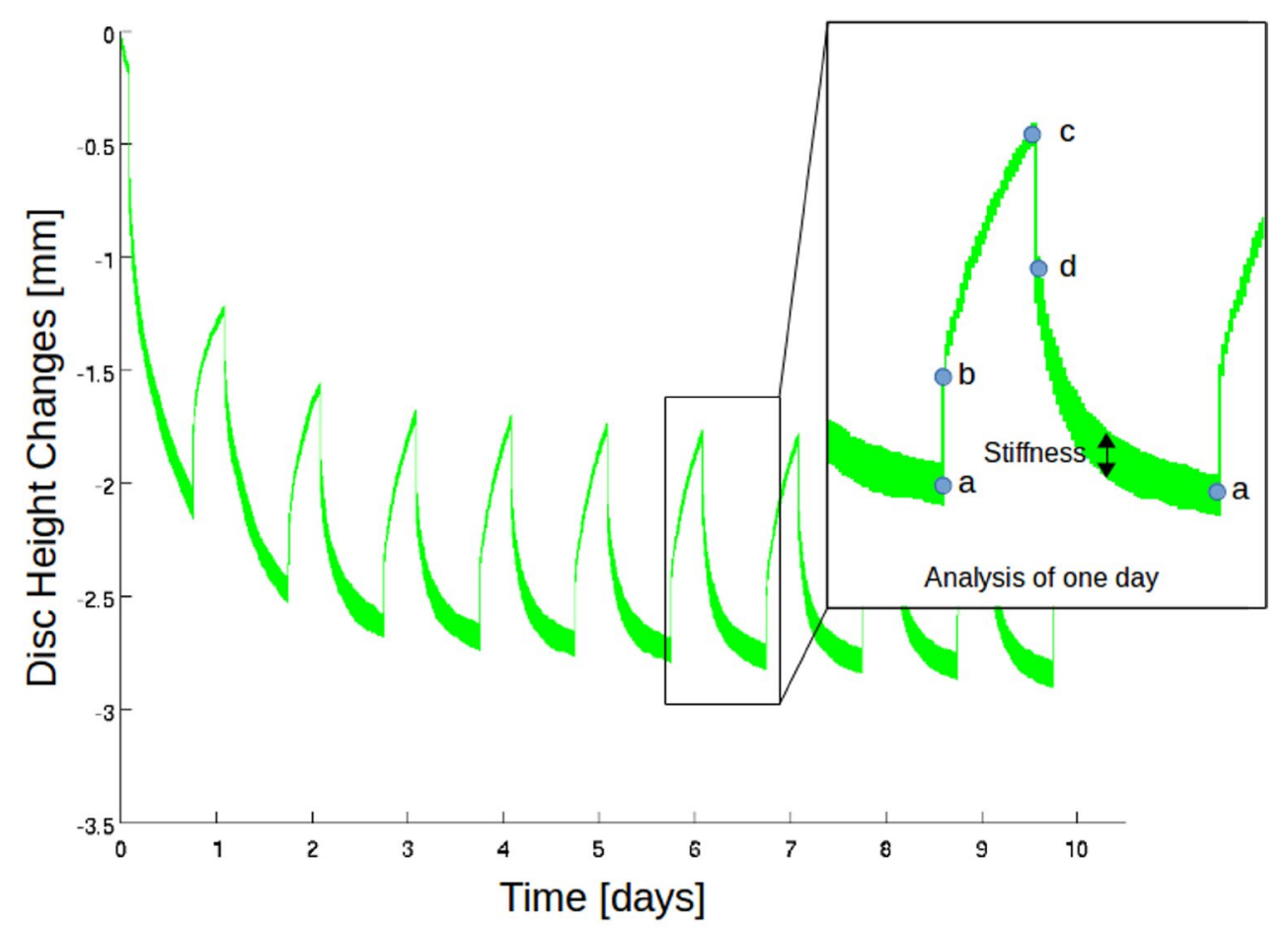

Fig. 3. Typical example of the response of an IVD to the applied load. When zoomed in at day 6 , the changes in disc height between point $\mathrm{a}$ and $\mathrm{b}$, and between $\mathrm{c}$ and $\mathrm{d}$ are instantaneous, elastic responses. The changes between $\mathrm{b}$ and $\mathrm{c}$, and between $\mathrm{d}$ and $\mathrm{a}$, are slow, poroelastic responses. Points a and $\mathrm{c}$ are defined as the average disc height during the final $50 \mathrm{~s}$ of the load phase, point $\mathrm{b}$ and $\mathrm{d}$ as the average disc height during the first $50 \mathrm{~s}$ of the load phase. The loss of disc height between point $d$ and a will be referred to as subsidence. The time constants and the stiffness are determined in the subsidence phase. The stiffness is calculated using the average amplitude in the subsidence phase. 
2001). Additionally, two alternative scores were obtained. The first method was introduced by Griffith et al. (2007), who suggested that the PS may be not discriminating enough in an elderly population, and adapted it to the Modified Pfirrmann Score (MPS), which has an eightpoint scale. The second method is the Thompson Score, a morphological five-point grading scale of a midsagittal slice (Thompson et al., 1990). This was graded on the basis of pictures obtained after the experiments described below were conducted.

From the obtained spines, all soft tissue and the posterior elements were removed, and IVDs were isolated by parallel cuts through the vertebral bone with a band saw. Approximately half a centimetre of bone was included to ensure inclusion of the endplate. The IVDs were submerged in PBS (Gibco ${ }^{\circledR}$ DPBS; Life Technologies, Carlsbad, CA, USA, osmolality of 280-320 mOsm $/ \mathrm{kg}$ ). To prevent infection, $10,000 \mu \mathrm{g} / \mathrm{mL}$ penicillin, $10 \mathrm{mg} / \mathrm{mL}$ streptomycin and $25 \mu \mathrm{g} / \mathrm{mL}$ amphoterizin B (PSF, Sigma-Aldrich ${ }^{\circledR}$, St. Louis, MO, USA) were added. As it was assumed that few cells in the IVD had survived freezing, no medium refreshments were conducted during the experiment. Discs were tested in a custom build culture system, previously described by Paul et al. $(2012,2013)$ and modified to allow testing of human specimens (Fig. 1).

After $2 \mathrm{~h}$ of preloading at $76 \mathrm{kPa}$, a diurnal loading regime was applied for $10 \mathrm{~d}$. We used $16 \mathrm{~h}$ of simulated daytime loading (sitting and walking), and $8 \mathrm{~h}$ of simulated night time loading (lying supine/prone). Applied pressures were based on in vivo measurements of intradiscal pressure during daily activities (Wilke et al., 1999). These intradiscal pressures were adjusted to axial compression pressures because previous in vitro compression studies showed intradiscal pressures in human IVDs to be 1.5 times the applied compressive pressure (Nachemson and Elfström, 1970). Therefore, all discs received a daily regime of $16 \mathrm{~h}$ of (average \pm sine amplitude) $370 \pm 130 \mathrm{kPa}$ and eight hours of $73 \pm 10 \mathrm{kPa}$ (Fig. 2). All loads were applied as a sine wave at a frequency of $1 \mathrm{~Hz}$.

\section{Data analysis}

Forces were measured with a Kam-e load cell (Bienfait, Haarlem, The Netherlands); disc height changes were measured with an OADM12 optoelectric sensor (Baumer, Berlin, Germany). Both signals were digitised at $100 \mathrm{~Hz}$. For analysis of the data, customised programs in Matlab (version 2012b for Linux, Mathworks, Natick, MA, USA) were used. Analysed outcome parameters include subsidence during daytime phases, time constants, and average stiffness per day (Fig. 3). Time constants of the axial deformation were calculated using the double-Voigt method, described by Van der Veen et al., (2007) using the long-term time constant. The quality of the fit was checked with visual inspection and linear regression analysis: only fits with a $\mathrm{R}^{2}>0.98$ with the original data were used for analysis. The rate of subsidence at the end of each day was obtained with a linear fit over the final half hour of the subsidence phase. The stiffness was calculated by dividing the amplitude of the force signal by the amplitude of the disc height signal, and averaging these ratios over all sine waves during the subsidence phase.

\section{Biochemical analysis}

Tissue samples for biochemical analysis of all discs were obtained from the nucleus pulposus. Samples were freeze-dried (Speedvac) and subsequently digested in a papain-digestion solution composed of a phosphate buffer, $100 \mathrm{mM}$ pH 6.5, 5 mM L-cysteine-HCl, 5 mM EDTA and $100 \mu \mathrm{g}$ papain per $\mathrm{mL}$ digestion solution (Merck Millipore, Billerica, MA, USA) overnight, in a water bath at $65^{\circ} \mathrm{C}$. Digested samples were diluted at will and GAG content was analysed using a dimethyl methylene blue (DMMB) assay (Biocolor Ltd, Carrickfergus, UK) according to manufacturer's protocol. This is a measure for proteoglycan content. Of the remaining papain-digestion solution, $500 \mu \mathrm{L}$ was used to quantify hydroxyproline (HYP) content, as a measure for total collagen content, using a 1,9 dimethylamino-benzaldehyde assay as described by Paul et al. (2012). Obtained nucleus GAG content was expressed in $\mu \mathrm{g}$ per $\mathrm{mg}$ tissue dry weight. Lastly, the nucleus GAG content was expressed relative to nucleus HYP content (GAG/HYP ratio), which has been shown to show strong changes with degeneration (Hoogendoorn et al., 2008). This is a measure for state of remodelling of the nucleus, as the nucleus cells change their extracellular matrix production with progressive degeneration from proteoglycan to collagen type-I, thus decreasing the GAG/ HYP ratio (Paul et al., 2013).

\section{Statistics}

To study the relation between several biomechanical parameters (i.e. subsidence, stiffness, time constant and rate of subsidence), degeneration gradings, biochemical measures, Spearman's rank correlation coefficient $(\rho)$ and corresponding $p$-values were calculated. Spearman's $\rho$ was used because of the ordinal nature of the grading data and the non-normal distribution of most biochemical data. Additionally, the Pfirrmann Score of the three observers were averaged and rounded to the nearest integer, after which a General Estimation Equation (GEE) with each spine as subject variable was used to pairwise compare consecutive Pfirrmann Score for each biomechanical parameter. Inter-observer consistency of the degeneration grading was determined using the averaged intra-class correlation coefficient. Furthermore, the parameters as obtained during day one of the experiment were compared to the parameters in equilibrium using a paired $t$-test. A Wilcoxon signed-rank test was performed to compare the pre-test Pfirrmann Score and the post-test Thompson Score.

\section{Results}

Of the 48 discs, 36 remained for analysis. Two were damaged during preparation and had to be discarded, and three discs were removed due to technical problems during measurements. One disc was removed, as no relevant disc height changes where measured, presumably due to clamping of the cover of the culture chamber. Six other discs were excluded because structural damage was observed during the experiments. Structural damage presented itself in the form of either sudden disc height changes (cracks) or an accelerating disc height loss at the 


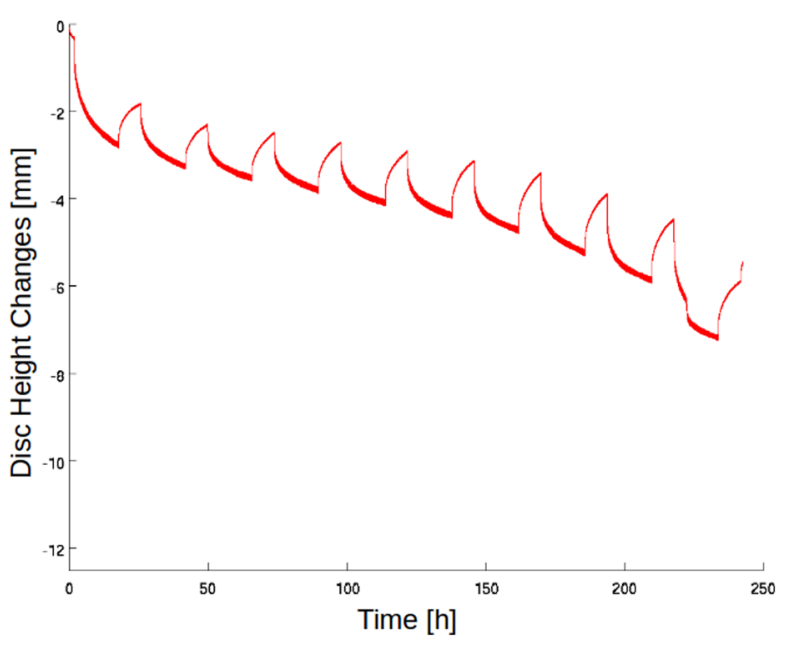

Fig. 4. Disc height changes of a discarded disc. Progressive reduction in disc height can be observed.

end of the experiment (accumulating damage) (Fig. 4). Six nuclei had to be excluded from biochemical analysis because not enough nucleus material could be identified, or the GAG content was too low to fit in the standard assay (less than $18 \mu \mathrm{g} / \mathrm{mg}$ dry weight).

The MRI grading of the 36 IVDs showed a distribution from mild to end-stage degeneration (PS 2-5, mean 3.39; MPS 2-8, mean 4.53; TS 2-5, mean 3.56). The averaged intra-class correlation coefficient between observers was 0.84 (PS), 0.87 (MPS) and 0.94 (TS). Fig. 5 shows a typical example of the mechanical response of a mildly and a severely degenerated disc. The first day shows great reduction in disc height, after which the discs enter a dynamic equilibrium after 4-5 d. Although the severely degenerated disc still loses disc height after day six, the parameters of interest do not change, which is the definition of equilibrium used in this study. For example, as can be seen in Fig. 6, no substantial changes in daily subsidence are found after day six. Therefore, the sixth day of loading was used for analysis, and compared with day one.

Table 1 shows that, except for the time constant, all parameters at day six differed significantly from day one. Correlations between MRI gradings and biomechanical parameters range from none to moderate. Notably, several parameters showed better correlation to the degeneration grades on day six than on the first day of loading. The PS correlated significantly with the subsidence at day six $(p=0.003)$, and the slope of the subsidence at the end of day one $(p=0.018)$ and six $(p=0.001)$. These parameters showed similar or slightly higher correlations with the MPS. The TS showed overall higher correlations, with only subsidence day one not reaching significance $(p=0.13)$. Except for the rate of subsidence at the end of day six, no significant relation between absolute nucleus GAG content and biomechanical parameters was found. If divided by a measure for total collagen content, the HYP content, significant relations were found with time constant $(p=0.008)$ and subsidence rate at the end of day

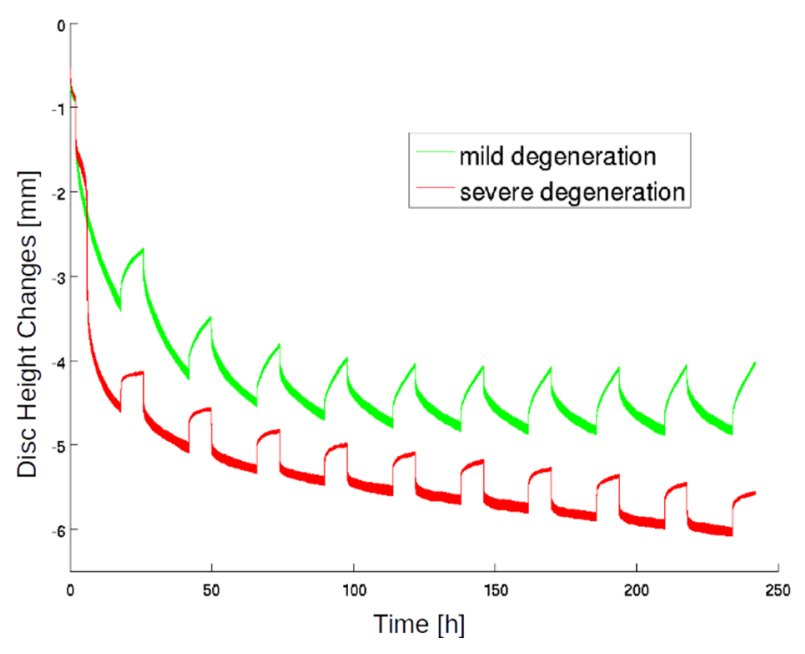

Fig. 5. Typical examples of the displacement graphs of an end-stage degenerated disc (PS 5) and a mildly degenerated disc (PS 3).

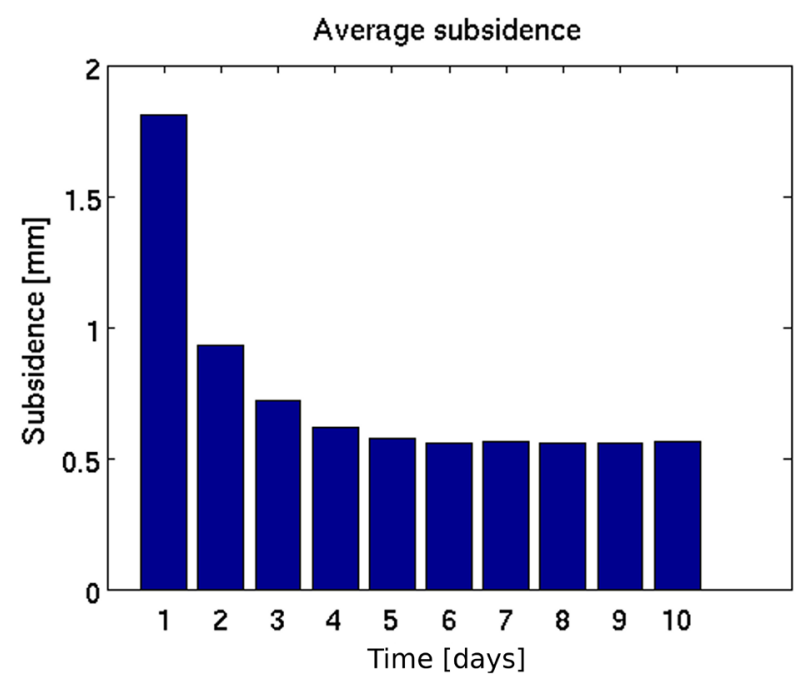

Fig. 6. Development of the daily subsidence over time. It can be seen that the parameter stabilises after $5 \mathrm{~d}$.

six $(p<0.001)$, and the stiffness at day one $(p=0.015)$. Overall, the GAG content seems to be a worse predictor of biomechanical properties than the GAG/HYP ratio (Fig. 7 ). It is noteworthy that the relation of the biomechanical parameters to GAG content and GAG/HYP ratio was consistently reverse in sign compared to the degeneration grades, as expected.

Fig. 8 shows the quantitative distribution over PSs of the parameters of interest on day six of the measurements. As shown, all parameters showed a highly significant main effect $(p<0.001)$, which indicates that correcting for the between-subject variability increases the statistical significance. Pairwise tests between consecutive PSs showed that the difference between grade 4 and 5 was the most notable in these parameters.

The Wilcoxon signed-rank test to compare pre-test PS and post-test TS did not show significant changes in degeneration $(p=0.42)$. 

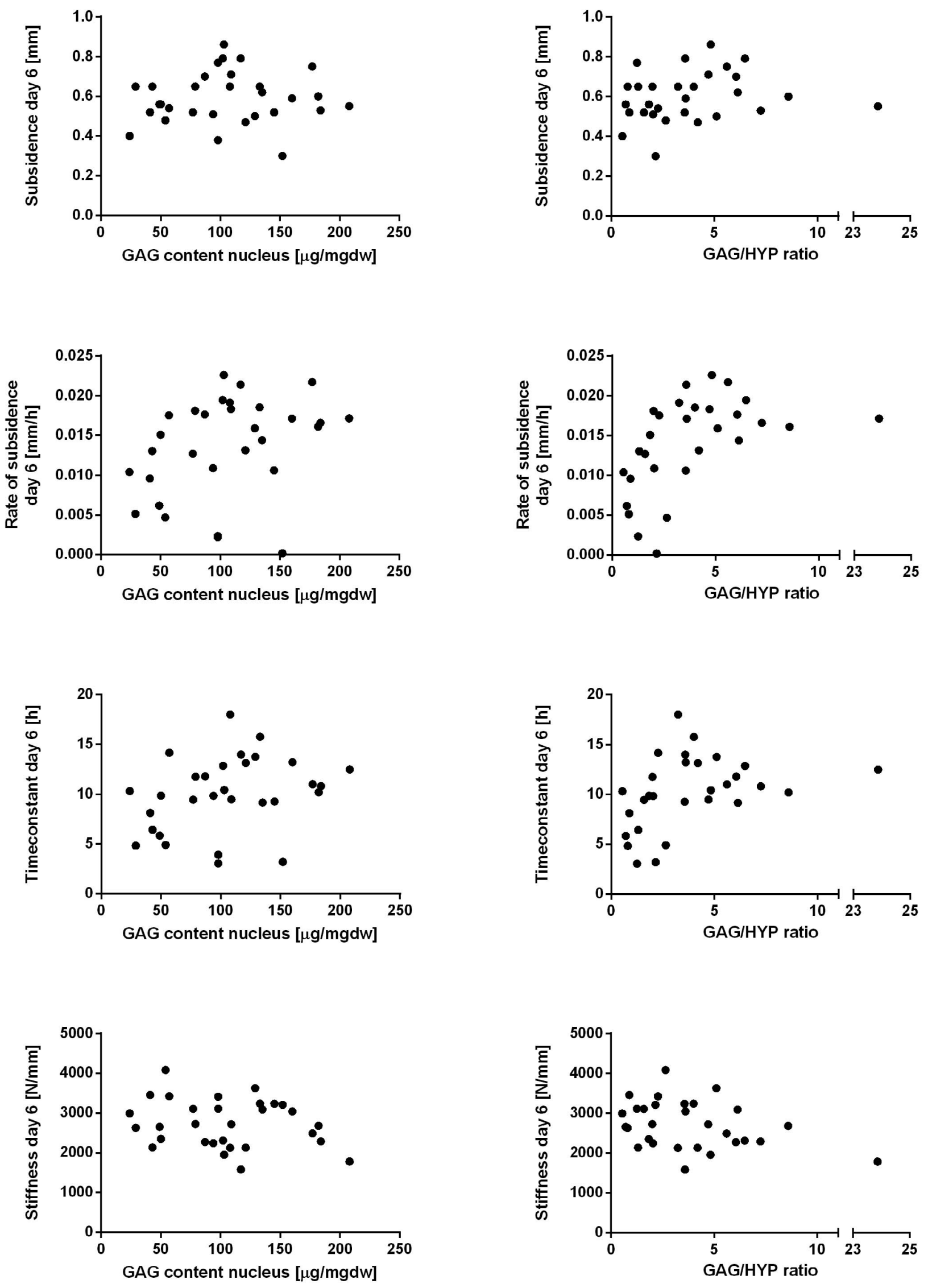

Fig. 7. Scatter plots of biochemical and biomechanical data. The biomechanical parameters are plotted on the left hand side against the GAG content, expressed in $\mu \mathrm{g}$ GAG per mg dry-weight of nucleus material $(\mu \mathrm{g} / \mathrm{mg}$ - $\mathrm{dw})$. On the right hand side, biomechanical parameters are plotted against the GAG content divided by the HYP content (GAG/HYP ratio). 
Table 1. Average values of the parameters and Spearman's correlation coefficients between biomechanical parameters, degeneration grades and biochemical parameters.

\begin{tabular}{|c|c|c|c|c|c|c|}
\hline Parameter & $\begin{array}{l}\text { Average value } \\
\text { (*significant } \\
\text { difference } \\
\text { from day 1) }\end{array}$ & $\begin{array}{c}\text { Correlation } \\
\text { with PS } \\
\end{array}$ & $\begin{array}{c}\text { Correlation } \\
\text { with MPS } \\
\end{array}$ & $\begin{array}{c}\text { Correlation } \\
\text { with TS } \\
\end{array}$ & $\begin{array}{c}\text { Correlation } \\
\text { with GAG- } \\
\text { content } \\
\text { nucleus } \\
\end{array}$ & $\begin{array}{c}\text { Correlation } \\
\text { with GAG/ } \\
\text { HYP ratio } \\
\text { nucleus }\end{array}$ \\
\hline Subsidence day 1 & $1.813 \mathrm{~mm}$ & -0.24 & -0.14 & 0.26 & -0.28 & -0.14 \\
\hline Subsidence day 6 & $0.559 \mathrm{~mm} * * *$ & $-0.49 * *$ & $-0.47 * *$ & $-0.33 *$ & 0.08 & 0.25 \\
\hline Rate of subsidence end day 1 & $0.043 \mathrm{~mm} / \mathrm{h}$ & $-0.39 *$ & $-0.50 * *$ & $-0.39 *$ & 0.16 & 0.31 \\
\hline Rate of subsidence end day 6 & $0.013 \mathrm{~mm} / \mathrm{h} * * *$ & $-0.53 * *$ & $-0.61 * * *$ & $-0.54 * * *$ & $0.37 *$ & $0.61 * * *$ \\
\hline Stiffness day 1 & $2489 \mathrm{~N} / \mathrm{mm}$ & $0.37 *$ & $0.44 * *$ & $0.59 * * *$ & -0.32 & $-0.45^{*}$ \\
\hline Stiffness day 6 & $2762 \mathrm{~N} / \mathrm{mm}^{* * *}$ & 0.30 & $0.45 * *$ & $0.52 * *$ & -0.14 & -0.27 \\
\hline Time constant day 1 & $11.0 \mathrm{~h}$ & -0.12 & -0.24 & $-0.54 * * *$ & 0.25 & 0.33 \\
\hline Time constant day 6 & $10.2 \mathrm{~h}$ & -0.27 & $-0.39 *$ & $-0.47 * *$ & 0.34 & $0.48 * *$ \\
\hline
\end{tabular}

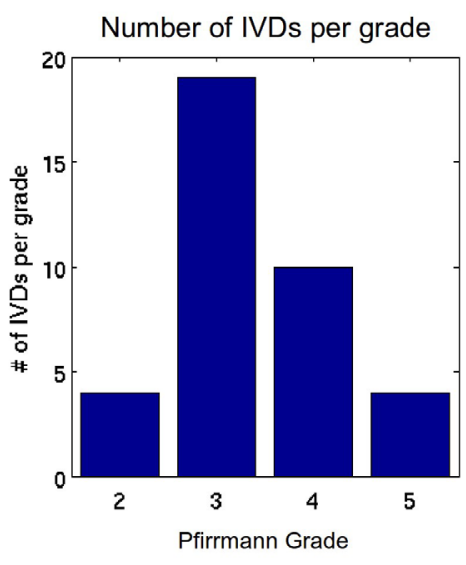

Fig. 8. Distributions over Pfirrmann grades. Top left: number of IVDs per PS group. Others: Average value for biomechanical parameters, grouped by PS. If main effect has been shown by the GEE, this is indicated after the title. Significance of the pairwise evaluation is shown in the graphs. ${ }^{*} p<0.05$, $* * p<0.01, * * * p<0.001$.
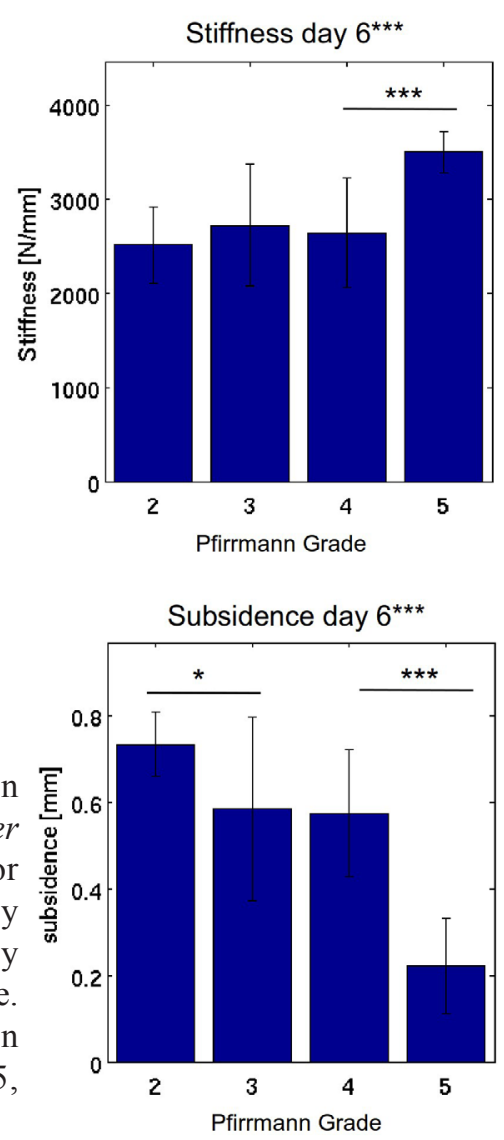
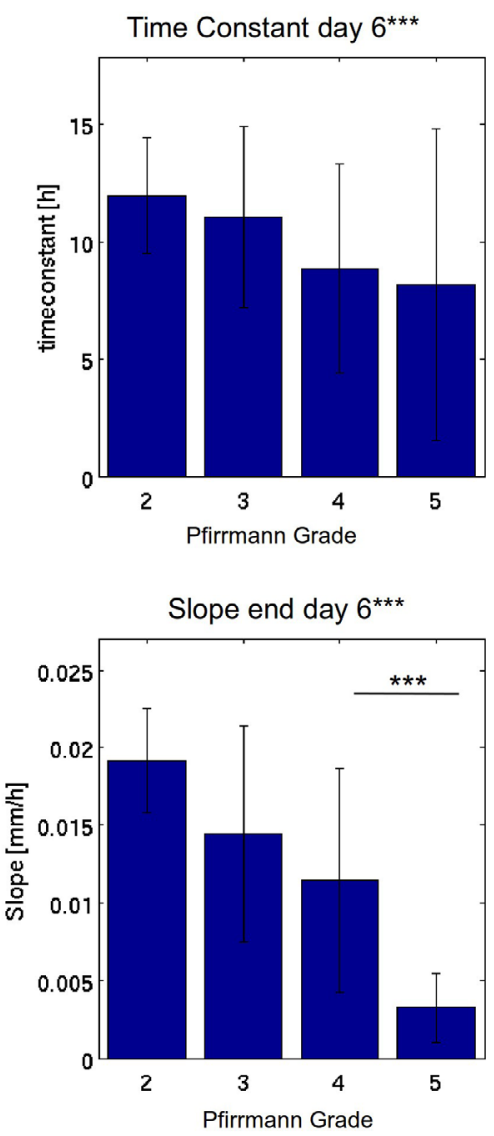

\section{Discussion}

This study was the first to measure biomechanical properties of entire human IVDs under an applied simulated physiological loading regime lasting ten days. Fig. 5 pre-eminently visualises the response of IVDs to the diurnal load, and how this changes with degeneration. It can be seen that the IVD shows instantaneous elastic response to changes in load, as well as slow poroelastic responses, in line with existing models (O'Connell et al., 2011; van der Veen et al., 2013). The results of this study indicate that degeneration of the IVD is related to (1) less subsidence during a day of loading, (2) lower subsidence rate at the end of the day, and (3) higher stiffness. The most pronounced differences were observed between IVDs with PS 4 and 5 , suggesting that biomechanical properties change most substantially during the end-stage degeneration.

Most of the parameters evaluated in this study were significantly different at day six of the experiment compared to day one. The subsidence during day six of the experiment showed a significant correlation with degeneration grade, but the subsidence at day one showed very weak, insignificant correlations. As shown in Fig. 6, the average subsidence per day reduced during the first five days, and was stable thereafter. In the first days of loading, the subsidence may have been related largely to the temporal state of hydration at the start of the experiment, which is difficult to control. This can be prevented by 
measuring multiple cycles of loading and unloading until parameters are stable. This study shows that the parameters then obtained may have a higher clinical relevance, because they show a better relation with IVD degeneration. The reason for this is that the initial condition of the IVDs was one of "superhydration" due to prolonged unloading after excision of the spinal segments. This means that the state of the disc was close to the maximal disc height, which, in vivo, would only occur after prolonged space-flight or bed-rest (O'Connell et al., 2011). Therefore, the amount of subsidence on the first day was consistently greater than the recovery during the first night, leading to a state of less hydration after day one, which reduced the possible amount of subsidence at subsequent days. After some days, the amount of subsidence and the recovery stabilised in a dynamic equilibrium. This was usually the case after six days.

Highest correlations with degeneration scores were found for the rate of subsidence at the end of each day. This indicates that with increasing degeneration, the subsidence is almost complete after a day of loading. The healthy discs, however, are still losing disc height at the end of the loading period. This shows that the healthy discs do not operate in the limits of the system, and maintain a dynamic behaviour, in contrast to degenerated discs.

The stiffness of the IVD in equilibrium did not show a significant correlation with PS, but did show significant correlations with MPS and TS. A main effect of PS on stiffness was found, however, in the GEE. In Fig. 8 it can be seen that the stiffness of the group of PS 4-5 deviates most pronouncedly, in line with other variables. A relation between stiffness or elastic modulus and degeneration has previously been found in the literature (Antoniou et al., 2013; Kaigle et al., 1998).

In contrast to the amount of subsidence itself, the time constant of the subsidence during day six did not show a significant relation with PS, although the GEE did show a main effect. However, with the TS, clear correlations with the time constant at day one and six are found. This may be partly explained by the fact that the time constants are rather large (average $10.2 \mathrm{~h}$ ) compared to the time measured (16 h). Van der Veen et al. (2013) recommend extending the measurement time at least 3 times the expected time constant to accurately acquire time constants. As the diurnal loading regime did not allow such an extension, estimations may be less robust, leading to more diffuse results. In future experiments, the final loading phase could be extended to approximately $60 \mathrm{~h}$ in order to estimate time constants more accurately.

The biomechanical parameters did not show a clear relation with GAG content in the nucleus. This is surprising, as proteoglycan loss is commonly accepted as the mediating factor in the relation between degeneration and biomechanics (e.g. Adams and Roughley, 2006; O'Connell et al., 2011). However, to our knowledge, only few studies have experimentally tested this relation. It has been established that enzymatic digestion of proteoglycan with injection of chondroitinase $\mathrm{ABC}$ (CABC) alters some mechanical parameters such as flexion mechanics
(Lü et al., 1997) and intradiscal pressure (Sasaki et al., 2001). In articular cartilage the proteoglycan content has been shown to correlate strongly with stiffness (Wheaton et al., 2005). Furthermore, nucleus pulposus material properties were found to be moderately related to GAG content (Johannessen and Elliott, 2005). Possibly, the contrasting findings in this study were limited by the specimen characteristics, as the GAG content in all nuclei was found to be low (maximal $208 \mu \mathrm{g} / \mathrm{mg}$ dry weight). The reason for this can be found in the age of the specimen, as GAG content is known to reduce with age, independent of degeneration (Singh et al., 2009). Six nuclei had to be excluded from analysis because not enough nucleus material could be identified, or the GAG content was too low to fit in the standard assay (less than $18 \mu \mathrm{g} / \mathrm{mg}$ dry weight). Together, this limited the range of GAG content, thereby probably reducing the possibility to find a strong correlation. The GAG content relative to the HYP content, however, did show significant moderate-tostrong correlations to time constant and subsidence rate. With degeneration, the cells in the nucleus produce less proteoglycans and start to produce more collagen type-I (Paul et al., 2013), which can be seen as remodelling of the disc. As HYP content is a measure for collagen content, the GAG/HYP ratio is an indicator for this process. The results of this study show that the remodelling of the nucleus from a proteoglycan-rich gel to a more collagen type-I-rich fibrous tissue is related to poroelastic properties of the disc. This indicates that besides the proteoglycans, the collagens also play a role in the poroelasticity of IVDs. However, to elucidate the relation between biochemical content and whole-segment biomechanical properties, more research is needed.

Altogether, measures for the slow, poroelastic response of the IVD to the increased load during daytime are significantly related to degeneration. These results support the common view on disc degeneration as a loss of capacity to bind water under load. This is reflected in the reduction of the poroelastic response. Presumably, this is caused by the reduced swelling capacity of the nucleus and/or the permeability of the system (i.e. IVD and endplates) for fluids. The swelling capacity of the nucleus is believed to be determined by the proteoglycan content (Urban and Roberts, 2003), although the results of this study imply that especially the ratio of proteoglycans and collagen may be of importance. The other possible mechanism that would influence poroelastic behaviour is an increase of the permeability of the system, which would disable the nucleus to build up pressure. The fluid flow out of the nucleus is known to be directed through the annulus rather than the endplate, as total blocking of the endplate does not influence biomechanical behaviour (Van der Veen et al., 2007). Furthermore, the permeability of the annulus is about tenfold higher than the permeability of the nucleus and endplate (Cortes et al., 2014). This indicates that the permeability of the annulus is not a main factor for the ability of the system to build up pressure, and that the permeability of the nucleus itself may be of more importance. Annulus fissures may therefore not necessarily 
have a direct effect on the axial biomechanics. The influence of annulus and nucleus permeability on wholedisc mechanics may be of interest for future research.

The number of discs that failed during this study (six) indicates that the loading was quite intense. A graph depicting one of the six discarded discs can be seen in Fig. 4. Damage of the subchondral bone was visually observed in this sample after the measurements. A possible explanation can be found in the old age of the donors at time of death, so that the subchondral bone was possibly osteoporotic. Furthermore, it is possible that the bone was weakened by sawing close to the endplate.

Freshly frozen human material was used in this study. Previous studies have shown that the biomechanical properties are not significantly altered after freezing and thawing human intervertebral discs compared to shortly post mortem (Dhillon et al., 2001; Tan and Uppuganti, 2012). Additionally, the frozen storage is expected to drastically lower the cell viability in the IVDs (Ohlendorf et al., 1996). Therefore, no active degeneration processes are expected. The TS, obtained after the experiments were conducted, did not show a significantly higher grading compared to the pre-test PS. Also, the stabilisation of parameters indicates that no on-going degenerative process is influencing the biomechanical parameters. The same applies to the biochemical content, where the number of viable cells that could actively break down the proteins is expected to be very low. In previous research no effect of freezing on GAG content was found (Guan et al., 2006).

An additional limitation may be found in the MRI images of the spines, as they were obtained post mortem. Therefore, the unloading of the spine prior to the MRI may have caused underestimation of the degeneration gradings in some discs, due to post mortem water uptake and subsequent increase in disc height. The non-significant difference between PS and TS implies that this is not of major influence, as the TS was obtained after the experiment, which normalises the state of superhydration.

\section{Implications}

The mechanical environment has great influence on IVD cell functioning. Both unloading and overloading have been shown to reduce cell viability, and alter gene expression (Paul et al., 2013). The reduction of poroelastic behaviour in degenerated discs implies a reduction in hydrostatic pressure. The reduction in hydrostatic pressure will increase the shear stresses in the IVD (Carter and Wong, 2003; Hwang et al., 2012), which is known to have a mechanobiological effect on the IVD cells. The cells respond to shear stress by activation of remodelling genes, which leads to increased production of collagen type-I (Carter and Wong, 2003, Paul et al., 2013). Furthermore, a reduction in hydrostatic pressure reduces proteoglycan production (Handa et al., 1997). This research showed that the ratio between proteoglycans and collagen is related to poroelastic properties of the disc. Possibly, this leads to a positive-feedback loop where degenerative mechanical cues induce remodelling, which causes more mechanical cues, etc. (Vergroesen et al., 2015a). Regenerative therapies should therefore aim to restore the biomechanical environment of the IVD cells, possibly by restoring the permeability and/or increasing swelling pressure through the water-binding capacity of the IVDs. For evaluation of disc-repair mechanisms and regenerative treatments, an exvivo environment like the loaded disc culture system could prove to be a useful tool, and the parameters identified in this study can be used to evaluate attempts to restore the functioning of the IVDs.

The results of this study indicate that the changing biomechanical properties of the disc show mostly a weak-to-moderate relation to degeneration, based on MRI imaging. End-stage degenerated discs (PS 5), although few in number, attributed most pronouncedly to this relation. The found absence of a strong relation could explain the difficulties encountered in finding a relation between MRI-based disc degeneration grading and low back pain, with the most convincing relations found in studies that specifically considered the most degenerated discs (Livshits et al., 2011). The accuracy of spinal degeneration assessment based on imaging can be compromised by the effects of loading history on disc fluid content. For instance, a $10 \%$ difference in MRI signal intensity has been reported between images taken in the morning and in the evening (Roberts et al., 1998). Additionally, it should be taken into account that the supine position of the patient will increase disc height instantaneously compared to standing due to the elastic response $(0.2-0.7 \mathrm{~mm}$ per disc in this study, see example in Fig. 3). Alternative imaging methods, such as the recently developed $\mathrm{T} 1_{\mathrm{p}}$ - and $\mathrm{T} 2{ }^{*}$-weighted MRI imaging techniques may provide stronger relations (Ellingson et al., 2014; Johannessen et al., 2006). However, new imaging techniques should be validated intensively to physiological parameters, before large and costly epidemiological studies are conducted. The Thompson Score shows overall stronger relation to biomechanical properties than the MRI-based degeneration scores. This indicates that this score is preferable in scientific context.

In conclusion, a loss of poroelastic behaviour of IVDs is related to the degeneration of the intervertebral disc, and its restoration should be a priority for attempts to regenerate discs. However, long-term loading is needed to obtain relevant measures. Furthermore, the Pfirrmann Score, which grades T2-weighted MRI images, is not a strong predictor of the biomechanical properties of IVDs. The ratio between proteoglycan content and collagen content (GAG/HYP ratio) showed stronger relations to disc biomechanics than solely the proteoglycan content. The relation between biochemical content and whole-disc mechanics needs further investigation.

\section{Acknowledgements}

The authors would like to thank Albert van der Veen, Paul Sinnige and Klaas Meyer for assistance in the preparation for the experiments; the toolshop of the VU University, Amsterdam, The Netherlands, for adapting the loaded disc culture system, and Otto Fabius for proofreading the manuscript. The authors acknowledge the support from ZonMW-VICI Grant 918.11.635, and the Dutch Arthritis Foundation. We wish to confirm that there are no known conflicts of interest associated with this publication and 
there has been no significant financial support for this work that could have influenced its outcome.

\section{References}

Adams MA, Roughley PJ (2006) What is intervertebral disc degeneration, and what causes it? Spine 31: 21512561.

Adams MA, Freeman BJ, Morrison HP, Nelson IW, Dolan P (2000) Mechanical initiation of intervertebral disc degeneration. Spine 25: 1625-1636.

Andersson GB (1999) Epidemiological features of chronic low-back pain. Lancet 354: 581-585.

Antoniou J, Epure LM, Michalek AJ, Grant MP, Iatridis JC, Mwale F (2013) Analysis of quantitative magnetic resonance imaging and biomechanical parameters on human discs with different grades of degeneration. J Magn Reson Imaging 38: 1402-1414.

Balagué F, Mannion AF, Pellisé F, Cedraschi C (2012) Non-specific low back pain - Authors' reply. Lancet 379: 1874-1875.

Brinckmann P, Grootenboer H (1991) Change of disc height, radial disc bulge, and intradiscal pressure from discectomy. An in vitro investigation on human lumbar discs. Spine 16: 641-646.

Carter DR, Wong M (2003) Modelling cartilage mechanobiology. Philos Trans R Soc Lond B Biol Sci 358: 1461-1471.

Chan SCW, Ferguson SJ, Gantenbein-Ritter B (2011) The effects of dynamic loading on the intervertebral disc. Eur Spine J 20: 1796-1812.

Cheung KMC, Karppinen J, Chan D, Ho DWH, Song Y-Q, Sham P, Cheah KSE, Leong JCY, Luk KDK (2009) Prevalence and pattern of lumbar magnetic resonance imaging changes in a population study of one thousand forty-three individuals. Spine 34: 934-940.

Cortes DH, Jacobs NT, DeLucca JF, Elliott DM (2014) Elastic, permeability and swelling properties of human intervertebral disc tissues: A benchmark for tissue engineering. J Biomech 47: 2088-2094.

Dhillon N, Bass EC, Lotz JC (2001) Effect of frozen storage on the creep behavior of human intervertebral discs. Spine 26: 883-888.

Ellingson AM, Nagel TM, Polly DW, Ellermann J, Nuckley DJ (2014) Quantitative T2* (T2 star) relaxation times predict site specific proteoglycan content and residual mechanics of the intervertebral disc throughout degeneration. J Orthop Res 32: 1083-1089.

Fry TR, Eurell JC, Johnson a L, Brown MD, Losonsky JM, Schaeffer DJ (1991) Radiographic and histologic effects of chondroitinase $\mathrm{ABC}$ on normal canine lumbar intervertebral disc. Spine 16: 816-819.

Griffith JF, Wang Y-XJ, Antonio GE, Choi KC, Yu A, Ahuja AT, Leung PC (2007) Modified Pfirrmann grading system for lumbar intervertebral disc degeneration. Spine 32: E708-712.

Guan J, Urban JPG, Li ZH, Ferguson DJP, Gong CY, Cui ZF (2006) Effects of rapid cooling on articular cartilage. Cryobiology 52: 430-439.
Handa T, Ishihara H, Ohshima H, Osada R, Tsuji H, Obata K (1997) Effects of hydrostatic pressure on matrix synthesis and matrix metalloproteinase production in the human lumbar intervertebral disc. Spine 22: 1085-1091.

Hoogendoorn RJ, Wuisman PI, Smit TH, Everts VE, Helder MN (2007) Experimental intervertebral disc degeneration induced by chondroitinase $\mathrm{ABC}$ in the goat. Spine 32: 1816-1825.

Hoogendoorn RJW, Helder MN, Kroeze RJ, Bank RA, Smit TH, Wuisman PIJM (2008) Reproducible long-term disc degeneration in a large animal model. Spine 33: 949954.

Hoy D, Bain C, Williams G, March L, Brooks P, Blyth F, Woolf A, Vos T, Buchbinder R (2012) A systematic review of the global prevalence of low back pain. Arthritis Rheum 64: 2028-2037.

Hsieh AH, Twomey JD (2010) Cellular mechanobiology of the intervertebral disc: new directions and approaches. J Biomech 43: 137-145.

Hwang D, Gabai AS, Yu M, Yew AG, Hsieh AH (2012) Role of load history in intervertebral disc mechanics and intradiscal pressure generation. Biomech Model Mechanobiol 11: 95-106.

Jim B, Steffen T, Moir J, Roughley P, Haglund L (2011) Development of an intact intervertebral disc organ culture system in which degeneration can be induced as a prelude to studying repair potential. Eur Spine J 20: 1244-1254.

Johannessen W, Elliott DM (2005) Effects of degeneration on the biphasic material properties of human nucleus pulposus in confined compression. Spine 30: E724-E729.

Johannessen W, Auerbach JD, Wheaton AJ, Kurji A, Borthakur A, Reddy R, Elliott DM (2006) Assessment of human disc degeneration and proteoglycan content using T1rho-weighted magnetic resonance imaging. Spine 31: 1253-1257.

Johnson EF, Chetty K, Moore IM, Stewart A, Jones W (1982) The distribution and arrangement of elastic fibres in the intervertebral disc of the adult human. J Anat 135: 301-309.

Kaigle A, Ekström L, Holm S, Rostedt M, Hansson $\mathrm{T}$ (1998) In vivo dynamic stiffness of the porcine lumbar spine exposed to cyclic loading: influence of load and degeneration. J Spinal Disord 11: 65-70.

Katz JN (2006) Lumbar disc disorders and low-back pain: socioeconomic factors and consequences. J Bone Joint Surg Am 88 Suppl 2: 21-24.

Kettler A, Wilke H-J (2006) Review of existing grading systems for cervical or lumbar disc and facet joint degeneration. Eur Spine J 15: 705-718.

Korecki CL, MacLean JJ, Iatridis JC (2007) Characterization of an in vitro intervertebral disc organ culture system. Eur Spine J 16: 1029-1037.

Lambeek LC, van Tulder MW, Swinkels ICS, Koppes LLJ, Anema JR, van Mechelen W (2011) The trend in total cost of back pain in The Netherlands in the period 2002 to 2007. Spine 36: 1050-1058.

Livshits G, Popham M, Malkin I, Sambrook PN, Macgregor AJ, Spector T, Williams FMK (2011) Lumbar disc degeneration and genetic factors are the main risk 
factors for low back pain in women: the UK Twin Spine Study. Ann Rheum Dis 70: 1740-1745.

Lü DS, Shono Y, Oda I, Abumi K, Kaneda K (1997) Effects of chondroitinase ABC and chymopapain on spinal motion segment biomechanics. An in vivo biomechanical, radiologic, and histologic canine study. Spine 22: 1828 1834.

Luoma K, Riihimäki H, Luukkonen R, Raininko R, Viikari-Juntura E, Lamminen A (2000) Low back pain in relation to lumbar disc degeneration. Spine 25: 487-492.

Nachemson A, Elfström G (1970) Intravital dynamic pressure measurements in lumbar discs. A study of common movements, maneuvers and exercises. Scand J Rehabil Med Suppl 1: 1-40.

O’Connell GD, Jacobs NT, Sen S, Vresilovic EJ, Elliott DM (2011) Axial creep loading and unloaded recovery of the human intervertebral disc and the effect of degeneration. J Mech Behav Biomed Mater 4: 933-942.

Ohlendorf C, Tomford WW, Mankin HJ (1996) Chondrocyte survival in cryopreserved osteochondral articular cartilage. J Orthop Res 14: 413-416.

Paul CPL, Zuiderbaan HA, Zandieh Doulabi B, van der Veen AJ, van de Ven PM, Smit TH, Helder MN, van Royen BJ, Mullender MG (2012) Simulated-physiological loading conditions preserve biological and mechanical properties of caprine lumbar intervertebral discs in ex vivo culture. PLoS One 7: e33147.

Paul CPL, Schoorl T, Zuiderbaan HA, Zandieh Doulabi B, van der Veen AJ, van de Ven PM, Smit TH, van Royen BJ, Helder MN, Mullender MG (2013) Dynamic and static overloading induce early degenerative processes in caprine lumbar intervertebral discs. PLoS One 8: e62411.

Pfirrmann CW, Metzdorf A, Zanetti M, Hodler J, Boos N (2001) Magnetic resonance classification of lumbar intervertebral disc degeneration. Spine 26: 1873-1878.

Roberts N, Hogg D, Whitehouse GH, Dangerfield P (1998) Quantitative analysis of diurnal variation in volume and water content of lumbar intervertebral discs. Clin Anat 11: $1-8$

Sasaki M, Takahashi T, Miyahara K, Hirose AT (2001) Effects of chondroitinase ABC on intradiscal pressure in sheep: an in vivo study. Spine 26: 463-468.

Sato K, Kikuchi S, Yonezawa T (1999) In vivo intradiscal pressure measurement in healthy individuals and in patients with ongoing back problems. Spine 24: 2468-2474.

Singh K, Masuda K, Thonar EJ-MA, An HS, CsSzabo G (2009) Age-related changes in the extracellular matrix of nucleus pulposus and anulus fibrosus of human intervertebral disc. Spine 34: 10-16.

Smit TH, Odgaard A, Schneider E (1997) Structure and function of vertebral trabecular bone. Spine 22: 2823-2833.

Tan JS, Uppuganti S (2012) Cumulative multiple freeze-thaw cycles and testing does not affect subsequent within-day variation in intervertebral flexibility of human cadaveric lumbosacral spine. Spine 37: E1238-1242.

Thompson JP, Pearce RH, Schechter MT, Adams ME, Tsang IK, Bishop PB (1990) Preliminary evaluation of a scheme for grading the gross morphology of the human intervertebral disc. Spine 15: 411-415.
Urban JP, Roberts S (2003) Degeneration of the intervertebral disc. Arthritis Res Ther 5: 120-130.

Urban JPG, Winlove CP (2007) Pathophysiology of the intervertebral disc and the challenges for MRI. J Magn Reson Imaging 25: 419-432.

Van der Veen AJ, van Dieën JH, Nadort A, Stam B, Smit TH (2007) Intervertebral disc recovery after dynamic or static loading in vitro: is there a role for the endplate? J Biomech 40: 2230-2235.

Van der Veen AJ, Bisschop A, Mullender MG, van Dieën JH (2013) Modelling creep behaviour of the human intervertebral disc. J Biomech 46: 2101-2103.

Van Tulder MW, Assendelft WJ, Koes BW, Bouter LM (1997) Spinal radiographic findings and nonspecific low back pain. A systematic review of observational studies. Spine 22: 427-434.

Vergroesen PPA, van der Veen AJ, van Royen BJ, Kingma I, Smit TH (2014) Intradiscal pressure depends on recent loading and correlates with disc height and compressive stiffness. Eur Spine J 23: 2359-2368.

Vergroesen PPA, Kingma I, Emanuel KS, Hoogendoorn RJ, Welting TJ, van Royen BJ, van Dieën JH, Smit TH (2015a) Mechanics and biology in intervertebral disc degeneration: a vicious circle. Osteoarthritis Cartilage doi: 10.1016/j.joca.2015.03.028.

Wang Y, Videman T, Battié MC (2012) Lumbar vertebral endplate lesions: associations with disc degeneration and back pain history. Spine 37: 1490-1496.

Wheaton AJ, Dodge GR, Elliott DM, Nicoll SB, Reddy R (2005) Quantification of cartilage biomechanical and biochemical properties via T1-rho magnetic resonance imaging. Magn Reson Med 54: 1087-1093.

Wilke HJ, Jungkunz B, Wenger K, Claes LE (1998) Spinal segment range of motion as a function of in vitro test conditions: effects of exposure period, accumulated cycles, angular-deformation rate, and moisture condition. Anat Rec 251: 15-19.

Wilke H, Neef P, Caimi M, Hoogland T, Claes LE (1999) New in vivo measurements of pressures in the intervertebral disc in daily life. Spine 24: 755-762.

\section{Discussion with Reviewers}

Reviewer II: How much do you expect post mortem swelling, as it is a function of degenerative grade, to affect the MRI parameters used in this study?

Authors: The influence of post mortem swelling may be two-fold. First, an increase in disc height is present, as the results of this study clearly indicate. Second, an increase of water content may occur. Combined, this will lead to an underestimation of the MRI grade. However, the postexperimental Thompson grading (which does not suffer from the superhydration, as the experiment removes this) did not significantly differ from the pre-test Pfirrmann Score, indicating that there was no great underestimation.

Reviewer II: How much of the poroelastic response of degenerated discs do you expect to depend on secondary permeability (arising from radial fissuring of the AF) as 
opposed to primary permeability and proteoglycan content of the tissue?

Authors: This is an interesting question. As the permeability of nucleus material is much lower than the permeability of healthy annulus material, we believe that annulus permeability is not the main determinant of the permeability of the system as a whole. However, as the annulus confines the nucleus material, the fissure may prohibit the disc to build up pressure. In our recent research (Vergroesen et al., 2015b) a puncture of the annulus did not affect biomechanical properties as much as we expected, except in discs where the nucleus herniated through the fissure. Therefore, we do not think that secondary permeability has a great direct influence on biomechanical behaviour.

\section{Additional Reference}

Vergroesen PPA, Emanuel KS, Sharifi S, Kingma I, Grijpma DW, Smit TH (2015b) A biodegradable glue for annulus closure: evaluation of strength and endurance. Spine 40: 622-628. 